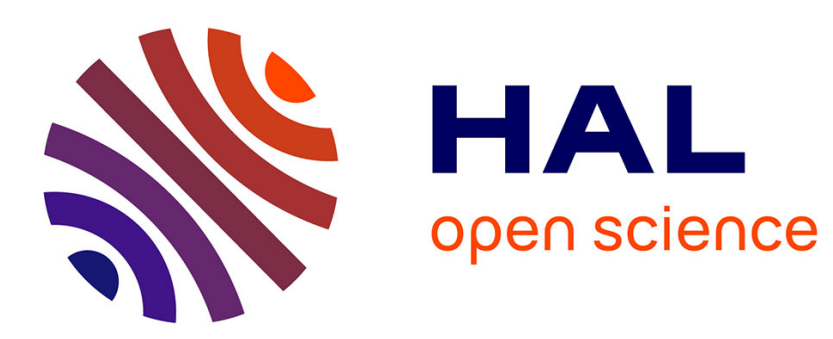

\title{
Calculation of the cross-plane thermal conductivity of a quantum cascade laser active region \\ M Szymaski
}

\section{To cite this version:}

M Szymaski. Calculation of the cross-plane thermal conductivity of a quantum cascade laser active region. Journal of Physics D: Applied Physics, 2011, 44 (8), pp.85101. 10.1088/00223727/44/8/085101 . hal-00597844

\section{HAL Id: hal-00597844 \\ https://hal.science/hal-00597844}

Submitted on 2 Jun 2011

HAL is a multi-disciplinary open access archive for the deposit and dissemination of scientific research documents, whether they are published or not. The documents may come from teaching and research institutions in France or abroad, or from public or private research centers.
L'archive ouverte pluridisciplinaire HAL, est destinée au dépôt et à la diffusion de documents scientifiques de niveau recherche, publiés ou non, émanant des établissements d'enseignement et de recherche français ou étrangers, des laboratoires publics ou privés. 


\title{
Calculation of the cross-plane thermal conductivity of a quantum cascade laser active region
}

\author{
M Szymański \\ Institute of Electron Technology, al. Lotników 32/46,02-668 Warsaw, Poland
}

\begin{abstract}
The key problem in thermal modelling of a quantum cascade laser is determining the thermal conductivity $\lambda$ of its active region. The parameter is highly anisotropic. Particularly the cross-plane value $\lambda_{\perp}$ is significantly reduced, which may be attributed to the presence of large number of interfaces between epitaxial layers. In this work two relatively simple models of phonon scattering at solid-solid boundary are used to calculate $\lambda_{\perp}$ for the terahertz quantum cascade laser. The theoretical results are in good agreement with measurements.
\end{abstract}

Keywords: terahertz quantum cascade laser (THz QCL), thermal boundary resistance, thermal conductivity, phonon scattering, superlattice

\section{Introduction}

Quantum-cascade lasers (QCLs) are semiconductor devices exploiting superlattices (SL's) as active layers. In numerous experimental works it has been shown that the thermal conductivity $\lambda$ of a superlattice is significantly reduced $[1,2,3]$. Particularly the cross-plane value $\lambda_{\perp}$ may be even order-of-magnitude smaller than than the value of constituent bulk materials. The phenomenon is a serious problem for QCLs, since they are electrically pumped by driving voltages over $10 \mathrm{~V}$ and current densities over $10 \mathrm{kA} / \mathrm{cm}^{2}$. Such a high injection power densities lead to intensive heat generation inside the devices. To make things worse, the main heat sources are located in the active layer, where the density of interfaces is the highest and - in consequence - the heat removal is obstructed. Thermal management in this case seems to be the key problem in design of the improved devices.

Theoretical description of heat flow across SL's is a really hard task. The crucial point is finding the relation between phonon mean free path $\Lambda$ and SL period $D$ [4]. In case $\Lambda>D$ both wave- and particle-like phonon behaviour is observed. The thermal conductivity is calculated through the modified phonon dispersion relation obtained from the equation of motion of atoms in the crystal lattice (see for example [5]). In case $\Lambda<D$ phonons behave like particles. The thermal conductivity is usually calculated using the Boltzmann transport equation with boundary conditions involving diffuse scattering. 
Unfortunately using the described methods in thermal model of QCL's is questionable. They are very complicated on the one hand and often do not provide satisfactionary results on the other. The comprehensive comparison of theoretical predictions with experiments for nanoscale heat transport one can find in Table II in [3]. This topic was also widely discussed in [6]. In addition, the investigations in this field usually deal with bilayer SL's, while one period of QCL active layer consists of dozen or so layers of order-of-magnitude thickness differences. In consequence mathematical models of heat flow in QCLs resemble these created for standard edge emitting lasers: they are based on heat conduction equation, isothermal condition at the bottom of the structure and convective cooling of the top and side walls are assumed. The SL is replaced by equivalent layer described by anisotropic values of thermal conductivity $\lambda_{\perp}$ and $\lambda_{\|}$, which are arbitrarily reduced [7, 8] or treated as fitting parameters [9]. Hence proposing a relatively simple method of assessing the thermal conductivity of QCL active region is necessary and is the goal of this work.

It is well known that the interface between materials of different thermal and mechanical properties disturbs the heat flow, introducing so called 'Kapitza resistance' or thermal boundary resistance (TBR) [10]. The phenomenon can be described by two phonon scattering models, namely the acoustic mismatch model (AMM) and the diffuse mismatch model (DMM). Input data are limited to such basic material parameters like Debye temperature, density or acoustic wave speed. Thus the thermal conductivity of the QCL active region can be calculated as a sum of weighted average of constituent bulk materials reduced by averaged TBR multiplied by the number of interfaces.

It is noteworthy, that similar approach using the DMM has been used once in thermal model of QCL [11]. However it has been done in the context of the temperature calculations of the entire device and the attention was focused rather on packaging details, while the problem of micro-scale heat transport was considered in the background.

In this paper the thermal conductivity of the terahertz QCL active layer is calculated by the AMM and DMM. The choice of the investigated structure followed from the possibility of comparison of theoretical results with values measured in [12] by the micro-probe band-to-band photo-luminescence. To become convinced about the reasonableness of the developed method, calculations for bilayer SiGe/SiGe SL of similar material composition has been provided and compared to values measured in [2]. The discussion of differences between two theoretical approaches is also included.

\section{The model}

The thermal conductivity of a multilayered structure can be approximated according to the rule of mixtures $[13,14]$ :

$$
\lambda^{-1}=\sum_{n} f_{n} \lambda_{n}^{-1}
$$


Table 1. List of symbols.

\begin{tabular}{|c|c|c|}
\hline Symbol & Units & Description \\
\hline$d_{n}$ & $\mathrm{~nm}$ & total thickness of the $n$-th medium \\
\hline$j=1,2,3$ & - & $\begin{array}{l}\text { phonon polarisation (one longitudinal-acoustic and two } \\
\text { transverse-acoustic) }\end{array}$ \\
\hline$k_{B}$ & $\mathrm{~J} / \mathrm{K}$ & Boltzmann constant \\
\hline$n=1,2$ & - & medium number \\
\hline$N$ & - & number of interfaces \\
\hline$r_{n}$ & $\mathrm{mK} / \mathrm{W}$ & thermal resistivity of the $n$-th material \\
\hline$r_{\mathrm{Bd}}(1 \rightarrow 2)$ & $\mathrm{m}^{2} \mathrm{~K} / \mathrm{W}$ & TBR for the heat flow from medium 1 to 2 \\
\hline$T$ & $\mathrm{~K}$ & temperature \\
\hline$\alpha_{1 \rightarrow 2}$ & - & $\begin{array}{l}\text { transmission probability for phonons propagating from } \\
\text { medium } 1 \text { to } 2\end{array}$ \\
\hline$\theta_{n}$ & $\mathrm{rad}$ & $\begin{array}{l}\text { angle between the phonon propagation direction in the } n \text {-th } \\
\text { medium and normal to the interface }\end{array}$ \\
\hline$\lambda_{\perp}, \lambda_{\|}$ & $\mathrm{W} / \mathrm{mK}$ & $\begin{array}{l}\text { thermal conductivity of QCL's active layer in the direction } \\
\text { perpendicular and parallel to epitaxial layers, respectively }\end{array}$ \\
\hline$\nu_{n, j}$ & $\mathrm{~m} / \mathrm{s}$ & $\begin{array}{l}\text { phonon velocity, subscripts } n \text { and } j \text { (if added) denote the } \\
\text { medium number and polarisation, respectively }\end{array}$ \\
\hline$\rho_{n}$ & $\mathrm{~kg} / \mathrm{m}^{3}$ & density, subscript $n$ (if added) denotes the medium number \\
\hline$\omega$ & $1 / \mathrm{s}$ & angular frequency \\
\hline$\omega_{D}=\frac{k_{B} \Theta_{D}}{\hbar}$ & $1 / \mathrm{s}$ & Debye frequency \\
\hline$\Theta_{D}$ & $\mathrm{~K}$ & Debye temperature \\
\hline
\end{tabular}

where $f_{n}$ and $\lambda_{n}$ are the volume fraction and bulk thermal conductivity of the $n$-th material. As it will be shown later (figure 3), in case of high density of interfaces the approach (1) is inaccurate. The influence of TBRs must be taken into account.

The active layer of a standard QCL is made of two materials epitaxially deposited in form of hundreds of alternate pairs. Thus its cross-plane thermal resistivity can be estimated as [11]

$$
\lambda_{\perp}^{-1}=\frac{d_{1}}{d_{1}+d_{2}} r_{1}+\frac{d_{2}}{d_{1}+d_{2}} r_{2}+\frac{N}{d_{1}+d_{2}} r_{\mathrm{Bd}}^{(\mathrm{av})},
$$

where TBR has been averaged with respect to the direction of the heat flow

$$
r_{\mathrm{Bd}}^{(\mathrm{av})}=\frac{r_{\mathrm{Bd}}(1 \rightarrow 2)+r_{\mathrm{Bd}}(2 \rightarrow 1)}{2}
$$

Description of symbols can be found in table 1 .

Now, determining how the heat flow from medium 1 to 2 is obstructed by TBR, is the crucial point of the analysis. According to [10]:

$$
r_{\mathrm{Bd}}=\left(\frac{1}{2} \sum_{j} \nu_{1, j} \Gamma_{1, j} \int_{0}^{\omega_{D}} \hbar \omega \frac{d N_{1, j}(\omega, T)}{d T} d \omega\right)^{-1},
$$


where

$$
\begin{aligned}
& N_{1, j}(\omega, T)=\frac{\omega^{2}}{2 \pi^{2} \nu_{1, j}^{3}\left[\exp \left(\hbar \omega / k_{B} T\right)-1\right]}, \\
& \Gamma_{1, j}=\int_{0}^{\pi / 2} \alpha_{1 \rightarrow 2}(\theta, j) \cos \theta \sin \theta d \theta .
\end{aligned}
$$

Calculation of phonon transmission probability between two solids is a real challenge. The problem has been considered in [15] via the Green's function method. However the described model is extremely complex and thus using simplified empirical approaches like AMM or DMM is desired.

In the AMM phonons are treated as plane waves propagating through continuum media (not through a lattice) [10]. The simplest version of the model neglects the mode conversion at the interface and assumes that velocities of all three polarisations are the same, which leads to [16]:

$$
\alpha_{1 \rightarrow 2}\left(\theta_{1}\right)=\frac{4 \rho_{1} \nu_{1} \rho_{2} \nu_{2} \cos \theta_{1} \cos \theta_{2}}{\left(\rho_{1} \nu_{1} \cos \theta_{1}+\rho_{2} \nu_{2} \cos \theta_{2}\right)^{2}}
$$

where $\theta_{1}$ and $\theta_{2}$ are related by Snell's law $\sin \theta_{1} / \nu_{1}=\sin \theta_{2} / \nu_{2}$. Considering the case $\nu_{1}>\nu_{2}$ these relations can be used without any restrictions. In the opposite case $\left(\nu_{1}<\nu_{2}\right)$ one should remember about the critical angle $\theta_{c}=\arcsin \left(\nu_{1} / \nu_{2}\right)$. Then, for $\theta_{1}<\theta_{c}$ the equation (7) is still valid, while for $\theta_{1}>\theta_{c}$ the phonon transmission probability vanishes, i.e. $\alpha_{1 \rightarrow 2}\left(\theta_{1}\right)=0$.

In the DMM it is assumed that phonon reaching the interface forgets where it came from and what was its polarisation. The probability of transmission does not depend on the incident angle and is determined by the mismatch between density of states in both media. This leads to [10]:

$$
\alpha_{1 \rightarrow 2}=\frac{\sum_{j} \nu_{2, j}^{-2}}{\sum_{n, j} \nu_{n, j}^{-2}} \text {. }
$$

\section{The results}

In this work I consider the THz QCL investigated experimentally in [12]. The active region consists of 90 periods of $\mathrm{GaAs} / \mathrm{Al}_{0.15} \mathrm{Ga}_{0.85} \mathrm{As}$. The layer thicknesses in one module, expressed in nanometers, are: 3.9/14.0/0.6/9.1/0.6/16.0/1.5/12.8/1.8/12.2/ 2.0/12.0/2.0/11.4/2.7/11.3/3.5/11.6. Bold font stands for AlGaAs barriers. The material parameters required for calculations are listed in table 2. The polynomial determining the Debye temperature for GaAs is the extrapolation of experimental data found in [17]. On the basis of [18] it has been assumed that $\Theta_{D}$ for $\mathrm{Al}_{0.15} \mathrm{Ga}_{0.85} \mathrm{As}$ is $2.7 \%$ higher than for GaAs.

The temperature dependence of TBR predicted by the AMM and DMM for GaAs $/ \mathrm{Al}_{0.15} \mathrm{Ga}_{0.85} \mathrm{As}$ interfaces is shown in figure 1 . The analysis of this plot provides the following findings consistent with other works: 


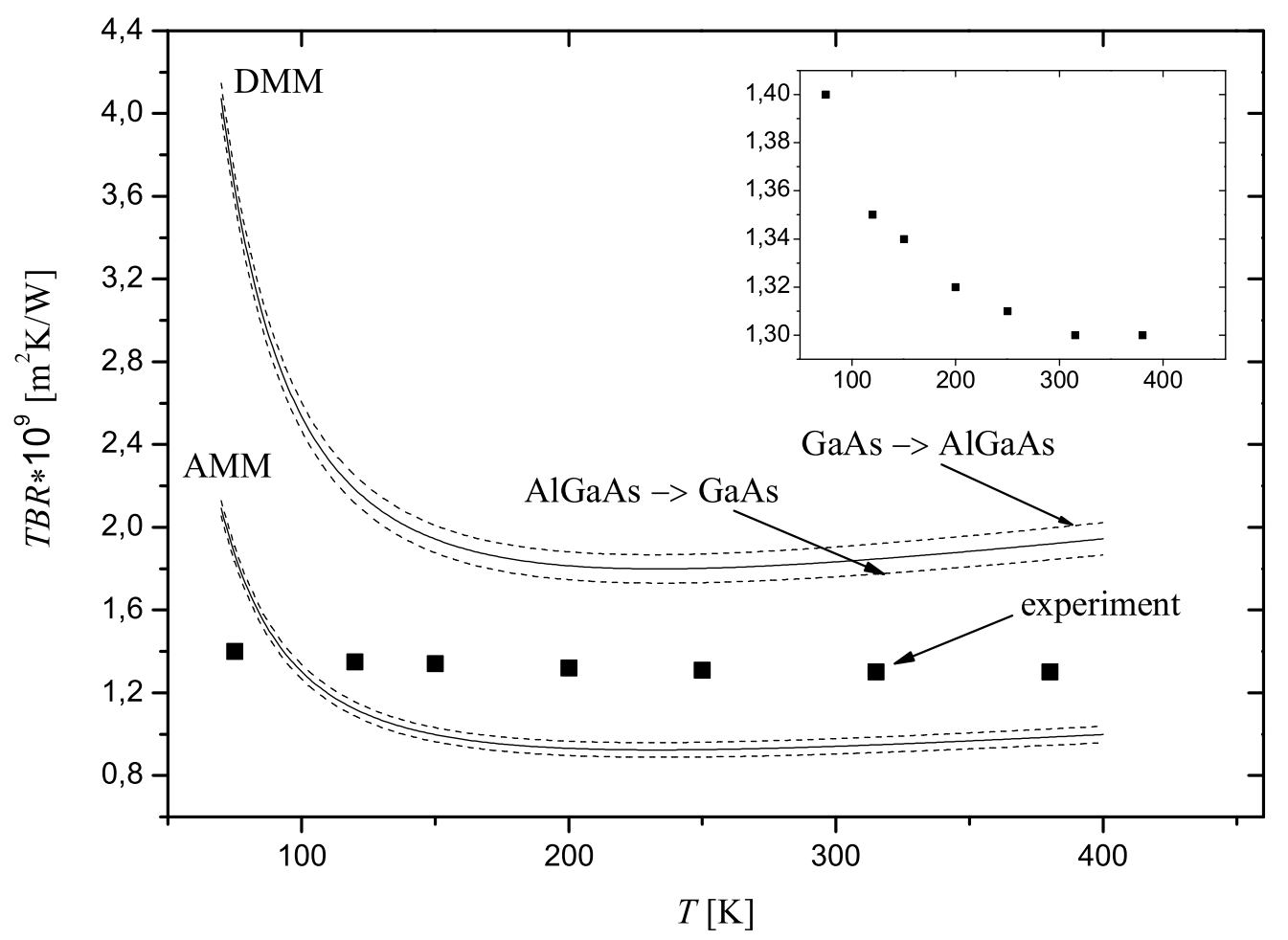

Figure 1. TBRs for GaAs $/ \mathrm{Al}_{0.15} \mathrm{Ga}_{0.85} \mathrm{As}$ system. Solid lines show the averaged values $r_{\mathrm{Bd}}^{(\mathrm{av})}$. Square symbols are the values measured in [12]. The inset with magnified scale shows that the increase of TBR with decreasing $T$ is observed experimentally.

Table 2. Material parameters [17, 18, 19].

\begin{tabular}{|l|l|l|l|}
\hline Parameter & $\mathrm{GaAs}$ & $\mathrm{Al}_{0.15} \mathrm{Ga}_{0.85} \mathrm{As}$ & $\mathrm{Si}_{1-x} \mathrm{Ge}_{x}(@ T=300 \mathrm{~K})$ \\
\hline$\Theta_{D}$ & $\begin{array}{l}377.64-0.14785 T+ \\
6.919 \cdot 10^{-5} T^{2}\end{array}$ & $1.027 \Theta_{D}(\mathrm{GaAs})$ & $640-266 x$ \\
\hline$\rho$ & $5320 \mathrm{~kg} / \mathrm{m}^{3}$ & $5086 \mathrm{~kg} / \mathrm{m}^{3}$ & $2329+3493 x-499 x^{2}$ \\
\hline$\nu(j=1)$ & $4730 \mathrm{~m} / \mathrm{s}$ & $4840 \mathrm{~m} / \mathrm{s}$ & $\sqrt{\frac{(165.8-37.3 x) \cdot 19^{9}}{\rho(x)}}$ \\
\hline$\nu(j=2,3)$ & $3350 \mathrm{~m} / \mathrm{s}$ & $3410 \mathrm{~m} / \mathrm{s}$ & $\sqrt{\frac{(79.6-12.8 x) \cdot 19^{9}}{\rho(x)}}$ \\
\hline$\lambda(T=300 \mathrm{~K})$ & $55 \mathrm{~W} / \mathrm{mK}$ & $28.8 \mathrm{~W} / \mathrm{mK}$ & $4.6+8.4 x$ \\
\hline$\lambda(T)$ & $\lambda(300 \mathrm{~K})\left(\frac{300 \mathrm{~K}}{T}\right)^{1.25}$ & $\lambda(300 \mathrm{~K})\left(\frac{300 \mathrm{~K}}{T}\right)^{1.25}$ & - \\
\hline
\end{tabular}

- the DMM predicts about 2 times larger value of TBR than the AMM, which is characteristic for interfaces between similar solid media (see figure 14 in Ref. [10]),

- there is a dramatic increase of TBR at low temperature [11],

- although TBR of single interface is rather low, the impact of interfaces on thermal 


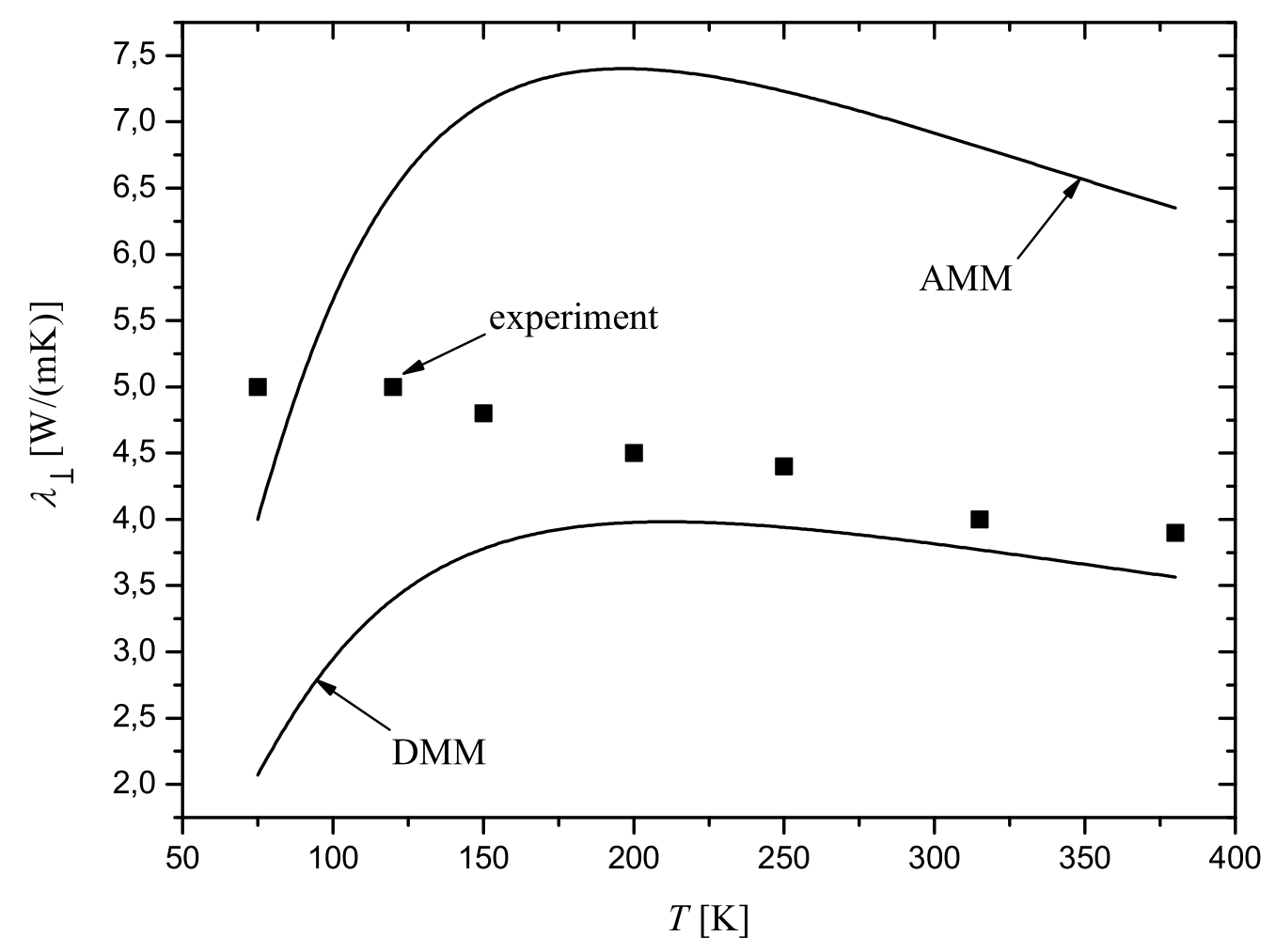

Figure 2. Calculated cross-plane thermal conductivity for the active region of $\mathrm{THz}$ QCL. Square symbols show the values measured in [12].

behaviour of QCL is significant due to their large number [11].

It is well seen (figure 1) that, within the wide range of temperature, the experimental values of TBR measured in [12] lie between those predicted by the DMM and AMM. The result can be explained after the detailed analysis of both models, which differ from each other by way of calculating the phonon transmission probability. A crucial assumption made in the AMM is that no scattering occurs at the interface [10]. In the DMM this assumption of complete specularity is replaced with the opposite extreme: all the phonons are scattered diffusely. However, empirically extrema occur rather seldom. In fact, any phonon scattering process can be treated as partially specular and partially diffuse process, which can be quantified by an interface specularity parameter $P$ [15]:

$$
\alpha_{1 \rightarrow 2}=P \alpha_{1 \rightarrow 2}^{\mathrm{AMM}}+(1-P) \alpha_{1 \rightarrow 2}^{\mathrm{DMM}},
$$

where $0 \leq P \leq 1$. The specularity varies of course with different interface parameters, like for example roughness. Thus the position of the measured TBRs with respect to calculated ones (figure 1) is the expression of such partially specular and partially diffuse nature of phonon scattering at interfaces. 
After considering TBRs of single interfaces, the cross-plane thermal conductivity $\lambda_{\perp}$ has been calculated according to equation (2) and plotted in figure 2. Note that the QCL active region consists of hundreds of interfaces, which are probably, from technological reasons, not ideal. Therefore assessing the value of $P$ is impossible and the presented calculations are based on purely specular (AMM) or diffuse (DMM) approaches.

Within the range of higher temperatures $(T>150 \mathrm{~K})$, better convergence between the experiment and calculations is found for the DMM than AMM. This suggests that phonons in the active layer are scattered rather diffusely than specularly, which may be a consequence of interface microroughness in the particular investigated sample. The presence of the 1-2 monolayer transition region at interfaces even in highquality GaAs/AlAs or GaAs/AlGaAs structures has been confirmed with the usage of postgrowth characterisation techniques [20, 21].

The discussion of results for the low temperatures is hindered with regard to modest number of works thoroughly focusing on this range. However it can be said, that two contrary phenomena influence the value of $\lambda_{\perp}$ while $T$ is decreasing: (i) the dramatic increase of TBR (please cf. figure 1) and (ii) the increase of bulk thermal conductivities according to formula's for $\lambda(T)$ given in table 2 and illustrated in figure 3 . The decreasing trend of $\lambda_{\perp}(T)$ in the low temperature limit (see figure 2) gives evidence of the superiority of (i). Here, the convergence with experiment described in [12] is slightly problematic. The experimental points with the last point measured for $T=75 \mathrm{~K}$ form rather a plateau than a slope. However measurements of SiGe/SiGe SL's carried out down to $50 \mathrm{~K}$ reveal the decreasing trend $\lambda_{\perp}(T)$ quite clearly [2].

Calculation of $\lambda_{\perp}$ according to the method proposed in this work is significantly simplified with respect to many other theoretical approaches (for example [4, 5]). In addition, it seems to be burdened by uncertainties of assessing $r_{\mathrm{Bd}}$, like averaging over the direction, unknown specularity $P$ of individual interface or assumptions underlying the AMM and DMM. To check its validity, $\lambda_{\perp}(T=300 K)$ has been calculated for the bilayer $\mathrm{Si}_{0.84} \mathrm{Ge}_{0.16} / \mathrm{Si}_{0.74} \mathrm{Ge}_{0.26}$ SL's. The choice of the structure followed from the possibility of comparison of theoretical results with values measured in [2], ease of finding material parameters for $T=300 \mathrm{~K}$ and similarity of constituent materials (like in the case of previously considered QCL active layer). Good convergence between the calculated and measured results is shown in table 3.

Table 3. Results for bilayer $\mathrm{Si}_{0.84} \mathrm{Ge}_{0.16} / \mathrm{Si}_{0.74} \mathrm{Ge}_{0.26} \mathrm{SL}$ 's. Note that due to experimental uncertainties $4.5<\lambda_{\perp}$ (measured) $<9.5$ (see Fig. 3 in [2]).

\begin{tabular}{|c|c|c|c|}
\hline SL period $[\mathrm{nm}]$ & $\lambda_{\perp}(A M M)$ & $\lambda_{\perp}(D M M)$ & $\lambda_{\perp}$ (measured) \\
\hline 10 & 5.4 & 3.7 & $\sim 7$ \\
\hline 15 & 6.6 & 4.8 & $\sim 7$ \\
\hline 20 & 7.5 & 5.6 & $\sim 7$ \\
\hline
\end{tabular}

The discussion of usefulness and exactness of the proposed method can be supplemented by figure 3, which clearly shows that neglecting the influence of interfaces 
leads to dramatic discrepancies between the calculated and measured values of the discussed parameter. Moreover one should keep in mind that the thermal behaviour of QCL depends on many factors or phenomena, which are difficult for quantitative analysis. Examples can be given easily from the thermal investigations of standard edge-emitting lasers:

- voids in the solder (such as inclusions of air, clean-up agents or fluxes) can weaken the thermal contact between the chip and heat spreader [22],

- the chip may not adhere to the heat sink entirely because of processing imperfections [23, 24],

- some of the researchers claim that convective cooling of the top and side walls plays a significant role [25], while others neglect this effect [26].

Hence, in my opinion, the accuracy of $\lambda_{\perp}$ calculated with the usage of the AMM, DMM or their combination expressed by equation (9) can be regarded as satisfactory.

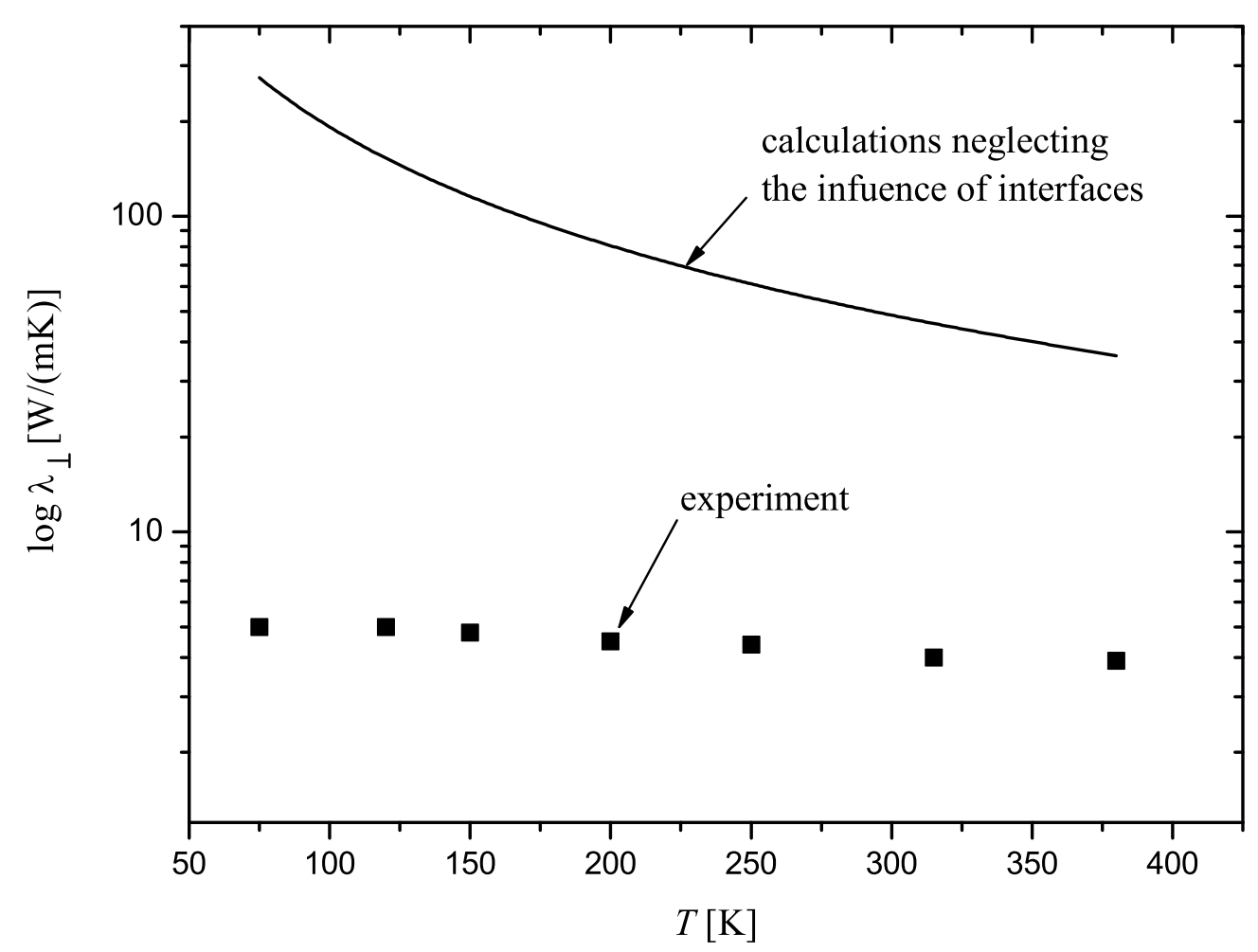

Figure 3. Illustration of large discrepancy between values of $\lambda_{\perp}$ measured in [12] and calculated according to equation (1), which neglects the influence of interfaces. 


\section{Conclusion}

In this paper two models of phonon scattering at solid-solid interfaces have been used to calculate the cross-plane thermal conductivity of active region of quantum cascade laser. The significant reduction of this parameter with respect to the bulk values of constituent materials has been confirmed. The obtained theoretical results are in good agreement with experiment. It is noteworthy that the presented approach is based on relatively simple calculations and requires the knowledge of usually easy available thermal or mechanical parameters of bulk materials. Therefore it can be a useful tool in thermal modelling of quantum cascade lasers.

\section{Acknowledgments}

The work was supported by the Polish Ministry of Science and Higher Education, grant No. PBZ-MNiSW02/I/2007. The author would like to thank Prof. Maciej Bugajski, Dr. Michał Wasiak and Dr. Anna Wójcik-Jedlińska for helpful discussions and critical reading of the manuscript.

\section{References}

[1] Capinski W S, Maris H J, Ruf T, Cardona M, Ploog K and Katzer D S 1999 Thermal-conductivity measurements of GaAs/AlAs superlattices using a picosecond optical pump-and-probe technique Phys. Rev. B 59 8105-8113

[2] Huxtable S T, Abramson A R, Chang-Lin T, and Majumdar A 2002 Thermal conductivity of $\mathrm{Si} / \mathrm{SiGe}$ and $\mathrm{SiGe/SiGe} \mathrm{superlattices} \mathrm{Appl.} \mathrm{Phys.} \mathrm{Lett.} 80$ 1737-1739

[3] Cahill D G, Ford W K, Goodson K E, Mahan G D, Majumdar A, Maris H J, Merlin R and Phillpot S R 2003 Nanoscale thermal transport J. Appl. Phys. 93 793-818

[4] Yang B and Chen G 2003 Partially coherent phonon heat conduction in superlattices Phys. Rev. B 67 195311-1-195311-4

[5] Tamura S, Tanaka Y and Maris H J 1999 Phonon group velocity and thermal conduction in superlattices Phys. Rev. B 60 2627-2630

[6] Gesikowska E and Nakwaski W 2008 An impact of multi-layered structures of modern optoelectronic devices on their thermal properties Opt. Quant. Electron. 40 205-216

[7] Lee H K, Chung K S, Yu J S and Razeghi M 2009 Thermal analysis of buried heterostructure quantum cascade lasers for long-wave-length infrared emission using 2D anisotropic heatdissipation model Phys. Status Solidi A, 206 356-362

[8] Evans C A, Jovanovic V D, Indjin D, Ikonic Z and Harrison P 2006 Investigation of thermal effects quantum cascade lasers IEEE J. Quantum Electron., 42 859-867

[9] Lops A, Spagnolo V and Scamarcio G 2006 Thermal modeling of GaInAs/AlInAs quantum cascade lasers J. Appl. Phys., 100 043109-1-043109-5

[10] Swartz E T and Pohl R O 1989 Thermal boundary resistance Rev. Mod. Phys. 61 605-668

[11] Zhu Ch, Zhang Y, Li A and Tian Z 2006 Analysis of key parameters affecting the thermal behaviour and performance of quantum cascade lasers J. Appl. Phys., 100 053105-1-053105-6

[12] Vitiello M S, Scamarcio G and Spagnolo V 2008 Temperature dependence of thermal conductivity and boundary resistance in THz quantum cascade lasers IEEE J. Sel. Top. in Quantum Electron., 14 431-435 
[13] Samvedi V and Tomar V 2009 The role of interface thermal boundary resistance in the overall thermal conductivity of Si-Ge multilayered structures Nanotechnology, 20365701

[14] Zhou Y, Anglin B and Strachan A 2007 Phonon thermal conductivity in nanolaminated composite metals via molecular dynamics J. Chem. Phys., 127184702

[15] Zhao H and Freund J B 2009 Phonon scattering at a rough interface between two fcc lattice J. Appl. Phys., 105 013515-1-013515-9

[16] Prasher R 2009 Acoustic mismatch model for thermal contact resistance of van der Waals contacts Appl. Phys. Lett., 94 041905-1-041905-3

[17] Glazov V M and Pashinkin A S 2000 Thermal expansion and heat capacity of GaAs and InAs Inorganic Mat., 36 225-231

[18] http://www.ioffe.ru/SVA/NSM/Semicond/

[19] Nakwaski W and Osiński M 1991 Thermal properties of etched-well surface-emitting semiconductor lasers IEEE J. Quantum Electron., 27 1391-1401

[20] Ruf T, Spitzer J, Sapega V F Belitzky V I Cardona M and Ploog K 1994 Interface roughness and homogeneous linewidths in quantum wells and superlattices studied by resonant acoustic-phonon Raman scattering Phys. Rev. B 50 1792-1806

[21] Jusserand B, Mollot F., Moison J and Le Roux G 1990 Atomic-scale roughness of GaAs/AlAs interface: A Raman scattering study of asymmetrical short-period superlattices Appl. Phys. Lett., 57 560-562

[22] Bärwolff A, Puchert R, Enders P, Menzel U and Ackermann D 1995 Analysis of thermal behaviour of high power semiconductor laser arrays by means of the finite element method (FEM) $J$. Thermal Analysis, 45 417-436

[23] Szymański M , Kozlowska A, Maląg A and Szerling A 2007 Two-dimensional model of heat flow in broad-area laser diode mounted to the non-ideal heat sink J. Phys. D: Appl. Phys., 40 924-929

[24] Lynch Jr. R T 1980 Effect of inhomogeneous bonding on output of injection lasers Appl. Phys. Lett. 36 505-506.

[25] Pipe K P and Ram R J 2003 Comprehensive Heat Exchange Model for a Semiconductor Laser Diode IEEE Photonic Technology Letters, 15 504-506.

[26] Romo G, Smy T, Walkey D and Reid B 2003 Modelling facet heating in ridge lasers Microelectronics Reliability 43 99-110 